\title{
Tiling Spaces of Taylor-Socolar Tilings
}

\begin{abstract}
J.-Y. LEE*
Department of Mathematics Education, Kwandong University, Gangneung, Gyeonggi-do, 210-701, Korea

The Taylor-Socolar tiling has been introduced as an aperiodic mono-tile tiling. We consider a tiling space which consists of all the tilings that are locally indistinguishable from a Taylor-Socolar tiling and study its structure. It turns out that there is a bijective map between the space of the Taylor-Socolar tilings and a compact Abelian group of a $Q$-adic space $(\bar{Q})$ except at a dense set of points of measure 0 in $\bar{Q}$. From this we can derive that the Taylor-Socolar tilings have quasicrystalline structures. We make a parity tiling from the Taylor-Socolar tiling identifying all the rotated versions of a tile in the Taylor-Socolar tiling by white tiles and all the reflected versions of the tile by gray tiles. It turns out that the Taylor-Socolar tiling is mutually locally derivable from this parity tiling.
\end{abstract}

DOI: 10.12693/APhysPolA.126.508

PACS: 61.44.Br, 61.44.-n

\section{Aperiodic mono-tile tilings}

The history of aperiodic tilings goes back to the time that Wang raised a question about whether there exists a set of finite prototiles which tile the plane only in aperiodic way. Some years later Berger found an example of an aperiodic tiling. Since then, many other aperiodic tilings have been found, for example, Robinson tiling, Ammann tiling, and Penrose tiling. Since Penrose found an aperiodic tiling with two prototiles, there has been a long search on finding a mono-tile which tiles a plane only in aperiodic way. As for the meaning of aperiodic mono-tile, there can be many ways of defining it depending on whether it allows to use the reflection of the tile or whether the tile should be a topological disk. Here allowing reflections of tiles but without the constraint of the topological disk of the tile, the Taylor-Socolar tile can be an example of an aperiodic mono-tile. The mono-tile of the Taylor-Socolar tiling is based on a hexagonal tile with certain matching rules which ensures aperiodic tilings (Fig. 1). The tile with the matching rules can be replaced by a single tile using only shape, making it a disconnected piece [1]. It can be reformed by a connected piece using cutpoints [2]. Yet it is not a topological disk. Nonetheless the fact that it is an aperiodic mono-tile opens up a new chapter on aperiodic tilings. Going back to the matching rule description, there are two basic rules:

(1) The black lines must join continuously when tiles abut.

(2) The ends of the diameters of two hexagonal tiles that are separated by an edge of another tile must be of opposite colors (Fig. 1).

When the plane is tiled with a given tile using these matching rules, the only possible tilings are the tilings

*e-mail: jylee@kwandong.ac.kr
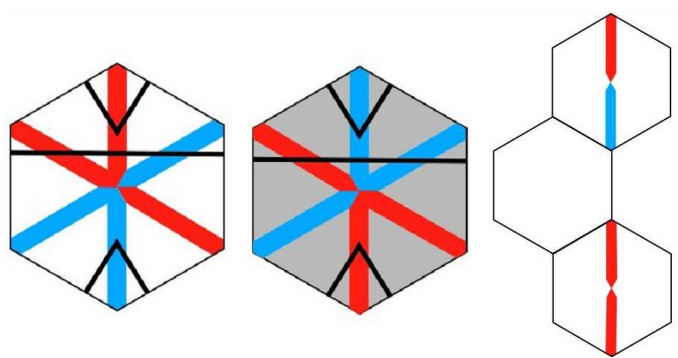

Fig. 1. The two tiles on the left hand side are the Taylor-Socolar tile and the reflected tile. The right hand side figure shows how the colored strips of tiles should meet.

which have a hierarchical structure of triangles made by the black strips as shown in Fig. 2. Due to the increasing size of the triangles, it is easy to observe that the tilings have to be aperiodic. This hierarchical structure of the triangles gives the main structure of the tiling. Then the matching rule on the colored stripes plays the role of fixing the hierarchical structure of triangles. In fact, without the matching rule on the colored stripes, it is possible to tile the plane by the tile periodically.

There is another aperiodic tiling built by single prototile using matching rules which were introduced by Penrose [3] (Fig. 3). The Penrose functional mono-tile tiling [3] arises from $\left(1+\epsilon+\epsilon^{2}\right)$-tiling [4,5]. This tiling is also based on hexagonal tiles as the Taylor-Socolar tiling. So it is a natural question to ask whether there is any relation between the Taylor-Socolar tiling and the Penrose functional mono-tile tiling. Is it possible to locally derive from one tiling to the other? The answer is negative, especially for non-generic tilings. In the 12th international conference on quasicrystals, Gähler suggested to consider a new tiling from which both of the Taylor-Socolar tiling and the Penrose functional mono-tile tiling can be locally derivable [6]. It has been studied in [7] how the two tilings are related and a new tiling from which both of the tilings can be locally derivable. 


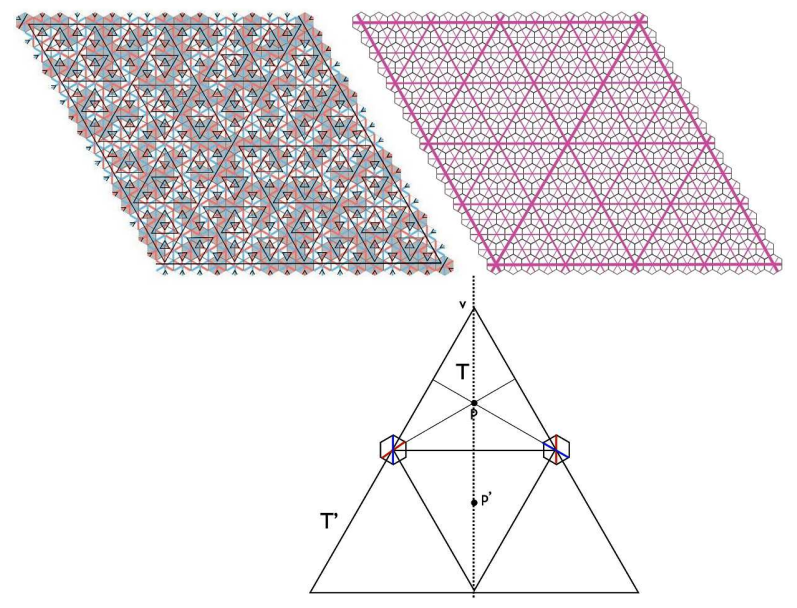

Fig. 2. The figure on the top left is a patch of a TaylorSocolar tiling. The top left right is a patch of a triangulation. The figure on the bottom shows how the colored strips of tiles determine.

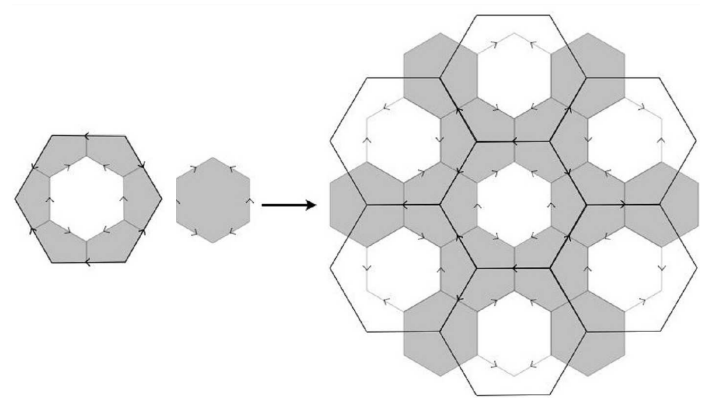

Fig. 3. The left hand side shows the mono-tile and the matching rule. The matching rule is that when two tiles meet at each vertex, the gray part should form a hexagon with the arrows on the edges as shown in the figure. A patch is given on the right.

\section{Tiling space of Taylor-Socolar tilings}

In order to understand the structural properties of the Taylor-Socolar tiling, we consider the tiling space of the Taylor-Socolar tilings and study the tiling dynamical system. Let $\mathcal{T}$ be a Taylor-Socolar tiling which is repetitive, that is to say, any patch in the tiling appears again and again within some bound. Let $X$ be the collection of all the tilings each patch of which is a translate of a $\mathcal{T}$-patch. We define a usual metric $d$ on $X$ : two tilings are close if they agree on a large region around the origin with a small shift [8]. Let $Q$ be the set of centers of the hexagonal tiles of the Taylor-Socolar tiling. We define $X_{Q}$ as the translational orbit closure of $\mathcal{T}$ using the metric $d$, i.e. $X_{Q}=\overline{\{-h+\mathcal{T}: h \in Q\}}$. We consider a group action of $Q$ on $X_{Q}$ by translations and get a topological dynamical system $\left(X_{Q}, Q\right)$.

As it is mentioned in Sect. 1, the hierarchical structure of triangles of black lines in the Taylor-Socolar tiling gives an important information of the structure of the tiling. So we will analyze this structure. Connecting the centers of the hexagonal tiles, a triangle lattice can be made on the hexagonal lattice tiling. We call this triangle lattice as 0-level triangle lattice. Then we choose the next level triangle lattice whose unit side length is twice bigger than the one in the previous level. We continue choosing the higher level triangle lattices in this manner and get the hierarchical structure of triangle lattices. On the other hand, the $Q$-adic topology on $Q$ is the uniform topology based on the metric defined by

$$
d(x, y)=2^{-k} \quad \text { if } x-y \in 2^{k} Q \backslash 2^{k+1} Q, \quad k \in \mathbb{Z}_{\geq 0} .
$$

Let $\bar{Q}$ be the completion of $Q$ in this topology. Then

$$
\begin{aligned}
& \bar{Q}=\left\{\left(q_{1}, q_{1}+q_{2}, \ldots, q_{1}+q_{2}+\ldots+q_{k}, \ldots\right) \mid\right. \\
& \left.\quad q_{k} \in 2^{k-1} Q \text { for all } k \in \mathbb{Z}_{+}\right\} .
\end{aligned}
$$

We can identify each construction of a triangulation on the hexagonal lattice with an infinite sequence

$$
\boldsymbol{q}=\left(q_{1}, q_{1}+q_{2}, \ldots, q_{1}+q_{2}+\ldots+q_{k}, \ldots\right) \in \bar{Q} .
$$

Now we describe what the colored stripes can determine on a triangulation for the construction of a tiling. Notice that both of the Taylor-Socolar tile and the reflected tile as shown on the left of Fig. 1 have long red and blue stripes in the same location of the tiles. So depending on where the half-red and half-blue stripes lie, tiles are completely determined. Once an $n$-level of a triangle in a triangulation is given as the top right figure of Fig. 2, two tiles on the centers of the edges of the $n$-level triangles should be given to match with the black lines of the tiles as shown in the bottom figure of Fig. 2. Then it determines all the tiles on the vertical lines of the edges of $n-1$ level triangles which lie on the edges on the $n$-level triangle except the centered $n-1$ level triangle. If we continue determining tiles in this way, we can determine most of tiles in the tiling (see the top two figures in Fig. 4).

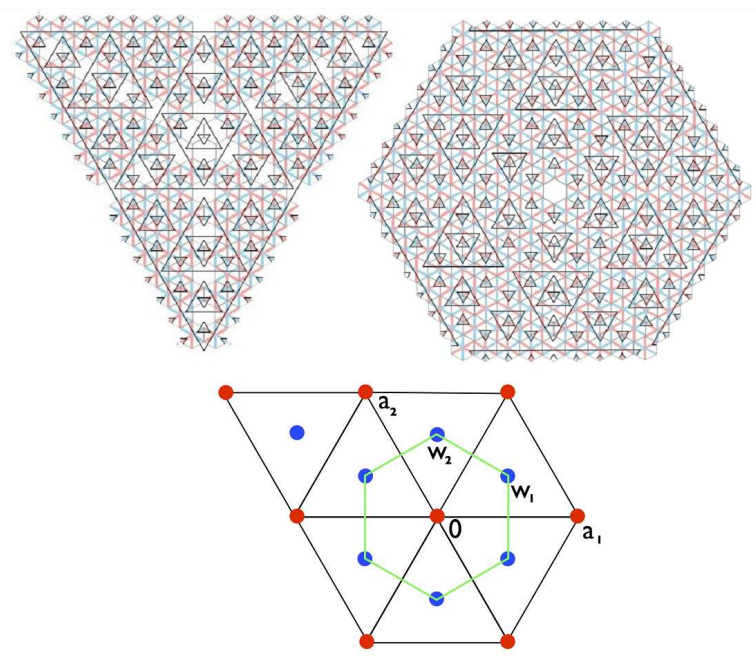

Fig. 4. The top two figures show the patterns of tilings whose tiles can be determined by the colored stripe rule from two non generic triangulations. The figure on the bottom indicates directional vectors to the vertexes and centers of hexagonal tiles in the tiling. 
Now we need to find out what determines the rest part of the tiling. For this, we need to classify the triangulations and define generic tilings in the tiling space. As shown in the bottom figure of Fig. 4 we give names on vectors in the hexagonal lattice and triangle lattice, where the hexagon centered at the origin corresponds to a hexagonal tile in the tiling. We call the lines of $a_{i^{-}}$ -direction a-lines and the lines of $w_{i}$-direction $w$-lines, where $a_{i} \in\left\{a_{1}, a_{2}, a_{1}+a_{2}\right\}$ and $w_{i} \in\left\{w_{1}, w_{2}, w_{2}-w_{1}\right\}$. We assign a level to each vertex of hexagonal tiles by the largest level of triangles in the triangulation which have the vertex as a center. Similarly we assign a level to each center point of hexagonal tiles by the largest level of triangles in the triangulation whose edges contain the center point. A triangulation is called generic- $w$ if every $w$-line has a finite level, i.e. for every $w$-line there is a finite bound on the levels of the centers that lie on that line. A triangulation is called generic- $a$ if every $a$-line has a finite level, i.e. for every $a$-line there is a finite bound on the levels of edges that lie on that line.
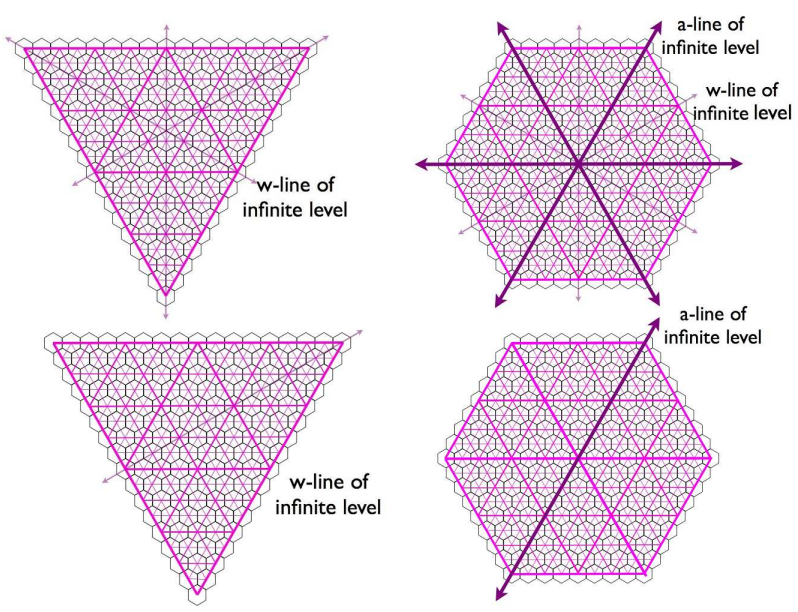

Fig. 5. The left of the top figures is a triangulation of generic- $a$ but not generic- $w$ tiling which is called $a i C w$ $L$ tiling. The right of the top figures is a triangulation of neither generic- $a$ nor generic- $w$ tiling which is called a CHT tiling. The left of the bottom figures is a triangulation of generic- $a$ but not generic- $w$ tiling. The right of the bottom figures is a triangulation of generic- $w$ but not generic- $a$ tiling.

There are three different types of non-generic triangulations: one type is generic- $a$ but not generic- $w$, another is generic- $w$ but not generic- $a$, and the other is neither generic- $w$ nor generic- $a$ (Fig. 5). We say that the tiling is generic- $a$ (or generic- $w$ ) if the triangulation is generic- $a$ (or generic- $w$ ). For the case of a generic tiling, the triangulation determines colored stripes of all the tiles in the tiling. For the case of a non-generic tiling, the triangulation cannot determine the tiling completely. For each type of non-generic tilings as given in Fig. 5, there are 6, 12 , and 2 possibilities of constructing the tilings. So we get the following theorem. Let $\xi$ be a mapping for which

$\xi: X_{Q} \rightarrow \bar{Q}$ by $\xi(\mathcal{T}) \mapsto \boldsymbol{q}(\mathcal{T})$, where $\boldsymbol{q}(\mathcal{T})$ is the corresponding infinite sequence in $\bar{Q}$ to $\mathcal{T}$ as described in (1).

Theorem 2.1 [9]. The mapping $\xi$ is continuous, surjective and 1-1 correspondence a.e. on $X_{Q}$. Furthermore $\xi$ is

(1) $1: 1$ on $X_{Q}^{\text {gen }}$;

(2) 6:1 at iCw-L tilings;

(3) 12:1 at CHT tilings;

(4) 2:1 at all other non-generic tilings.

This theorem shows that the dynamical spectrum of $\left(X_{Q}, Q\right)$ is isomorphic to the dynamical spectrum of a compact Abelian group $(\bar{Q}, Q)$. So mathematical theory tells us that the Taylor-Socolar tilings have the structures of quasicrystals.

\section{Parity tilings}

We construct a parity tiling from a Taylor-Socolar tiling removing all the decorative information on the tiles and only leaving out the locational information of the Taylor-Socolar tiles and the reflected tiles in the tiling (see the left figure of Fig. 6). A question is whether the Taylor-Socolar tiling is locally derivable from the parity tilings or not. Since a lot of information about the Taylor-Socolar tiling is lost in this case, it is hard to expect that at the first sight. But surprisingly the parity tiling keeps the structural information of the tiling and it is possible to reconstruct the original Taylor-Socolar tiling back.

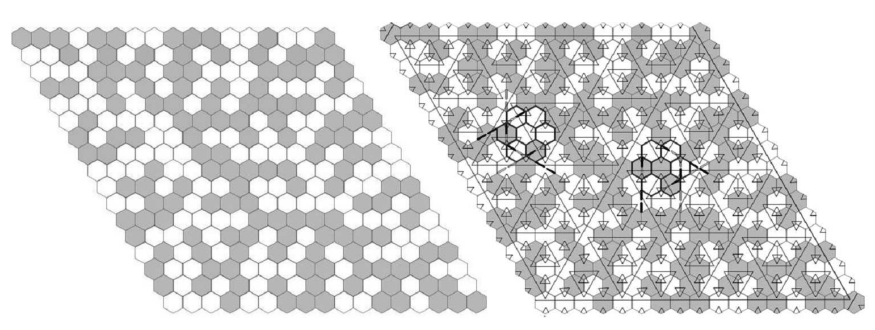

Fig. 6. The parity tiling on the left. The right figure shows the patterns of surrounding tiles of the corner tiles of 1 or higher level triangles.

Here we describe how it can be done. First we need to note the patterns of surrounding tiles of corner tiles of 1 or higher level triangles as shown in the right hand side of Fig. 6. Then the two patterns shown on the top of Fig. 7 cannot occur around the corner tiles of triangles (level $\geq 1$ ). From this observation we can try to find the location of the first level triangles in the parity tiling (see the two lower figures of Fig. 7). Once the location of the first level of triangles is determined, we build the next level hexagonal tiling. Then we try to find the location of the second level triangles in the new hexagonal tiling and get the third level hexagonal tiling (see the top 


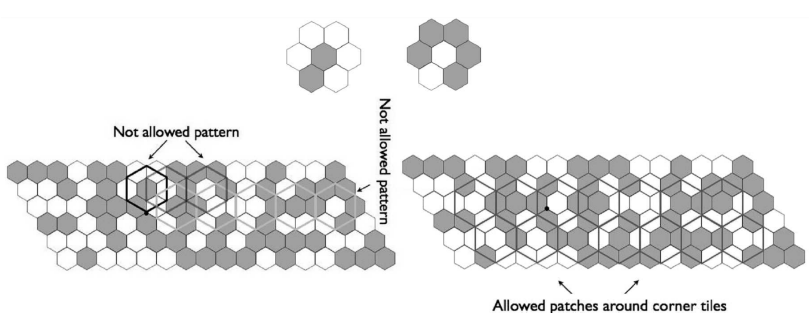

Fig. 7. The two patterns on the top are forbidden patterns as the patterns of surrounding tiles of the corner tiles of 1 or higher level triangles. A pattern on the lower left shows the wrong choices of the corner tiles of 1 or higher level triangles and a pattern on the right shows the right choice.

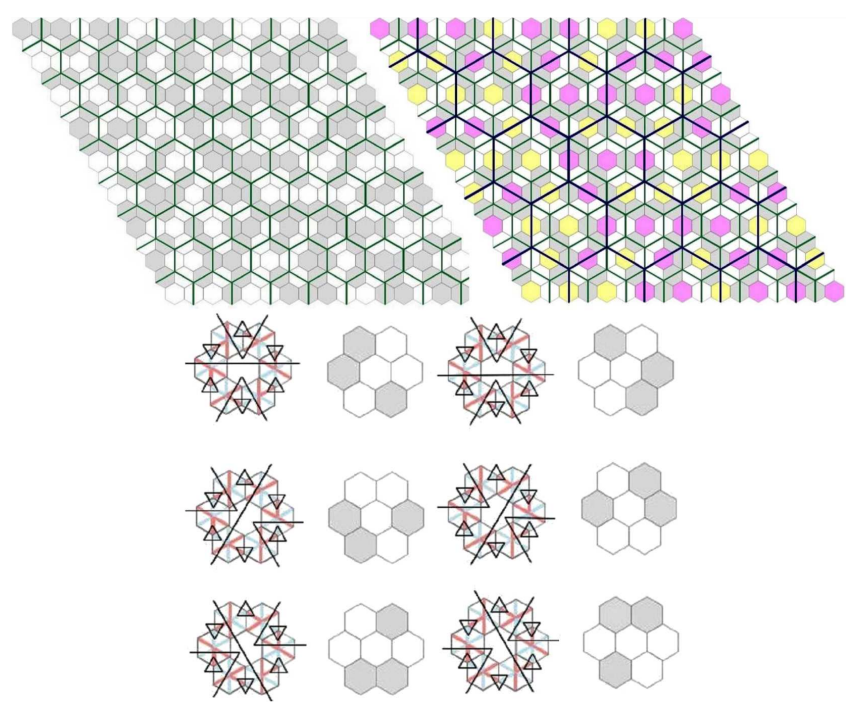

Fig. 8. The top two figures show how to build the second and third level hexagonal tilings in the parity tiling. The bottom figure shows the corresponding Taylor-Socolar tiling patterns to the partity patterns surrounding the corner tiles of triangles of second or higher levels.

two figures in Fig. 8). We do not need to continue this process to reconstruct the Taylor-Socolar tiling. Instead we do determine what types of patterns of the TaylorSocolar tiling can fit into the third level hexagonal tiles. So we first try to find the patterns of 7 hexagonal tiles around the center tiles in the third level hexagons. Before that, we need to notice what kind of patterns are possible around the corner tiles of 2 or higher level triangles from the Taylor-Socolar tiling. Then only 12 different patterns are possible and each one of them can be identified with exactly one pattern from the parity tiling (see the bottom part of Fig. 8). Now we replace the patterns of 7 hexagonal tiles in the parity tiling of the top right part of Fig. 8 by the corresponding patterns of 7 hexagonal tiles of Taylor-Socolar tiling shown in the bottom part of Fig. 8. Then using the match rule that the black lines have to be connected, we can complete the reconstruction of the Taylor-Socolar tiling. So a Taylor-Socolar tiling is locally derivable from a parity tiling. Since a parity tiling is locally derivable from a Taylor-Socolar tiling trivially, we get the following theorem.

Theorem 3.1 [9] A Taylor-Socolar tiling is mutually locally derivable from a parity tiling.

It is still a remaining question if there is a genuine single prototile allowing only translations, but not reflections, which tiles the plane only aperiodically.

\section{Acknowledgments}

The author is grateful for the helpful discussion to Michael Baake and Franz Gähler, and for the support of Basic Research Program through the National Research Foundation of Korea (NRF) funded by the Ministry of Education, Science and Technology (2010-0011150) as well as the support of KIAS.

\section{References}

[1] J. Socolar, J. Taylor, J. Combinat. Theor., Series A 118, 2207 (2011).

[2] J. Socolar, J. Taylor, Math. Intelligencer 34, 18 (2012).

[3] R. Penrose, Twistor Newsletter, reprinted in Roger Penrose: Collected Works, Vol. 6, 1997-2003, Oxford University Press, New York 2010.

[4] R. Penrose, in: The Mathematics of Long-Range Aperiodic Order, Ed. R.V. Moody, NATO ASI Series C, Vol. 489, Kluwer Acad. Publ., Dordrecht (Netherlands) 1997, p. 467.

[5] M. Baake, F. Gähler, U. Grimm, Symmetry 4, 581 (2012).

[6] F. Gähler, private communication.

[7] J.-Y. Lee, R.V. Moody, "A Relation between Two Aperiodic Hexagonal Tilings", preprint.

[8] C. Radin, M. Wolff, Geometr. Dedic. 42, 355 (1992).

[9] J.-Y. Lee, R.V. Moody, Symmetry 5, 1 (2013). 